ANNALES

POLONICI MATHEMATICI

$80(2003)$

\title{
Regularity of domains of parameterized families of closed linear operators
}

\author{
by Teresa Winiarska and Tadeusz Winiarski (Kraków)
}

To Professor Józef Siciak on the occasion of his 70th birthday

\begin{abstract}
The purpose of this paper is to provide a method of reduction of some problems concerning families $A_{t}=(A(t))_{t \in \mathcal{T}}$ of linear operators with domains $\left(\mathcal{D}_{t}\right)_{t \in \mathcal{T}}$ to a problem in which all the operators have the same domain $\mathcal{D}$. To do it we propose to construct a family $\left(\Psi_{t}\right)_{t \in \mathcal{T}}$ of automorphisms of a given Banach space $X$ having two properties: (i) the mapping $t \mapsto \Psi_{t}$ is sufficiently regular and (ii) $\Psi_{t}(\mathcal{D})=\mathcal{D}_{t}$ for $t \in \mathcal{T}$. Three effective constructions are presented: for elliptic operators of second order with the Robin boundary condition with a parameter; for operators in a Hilbert space for which eigenspaces form a complete orthogonal system of closed linear subspaces; and for a class of closed operators having bounded inverses.
\end{abstract}

1. Introduction. Most of the results concerning differential operators with a parameter $t$ in the coefficients have been obtained under the assumption that the operators $\left(A_{t}=A(t)\right)_{t \in \mathcal{T}}$ of a given family have domains independent of $t$ (see e.g. $[2,6,7,8]$ ).

One of possible ways of handling some problems concerning operators $\left(A_{t}\right)_{t \in \mathcal{T}}$ with domains $\mathcal{D}_{t} \subset X$ depending on $t$ is to find a sufficiently regular (with respect to $t \in \mathcal{T}$ ) family $\Psi_{t}$ of automorphisms of the Banach space $X$ such that $\Psi_{t}\left(\mathcal{D}_{t}\right)=\mathcal{D}$, where $\mathcal{D}$ is a fixed linear subspace of $X$.

In general, the domain of a differential operator is determined by some boundary conditions. Thus it would be useful to find an effective construction of a family $\Psi_{t}$ using the boundary conditions only. Such a construction for a family of elliptic operators of order two with the Robin boundary condition with a parameter (i.e. $\partial u / \partial n+a(x, t) u=0$ on $\partial \Omega$ ) is presented in 2.1. The problem of existence and construction of a family $\left(\Psi_{t}\right)_{t \in \mathcal{T}}$ for general types of boundary conditions is more delicate and still open.

2000 Mathematics Subject Classification: 47A05, 35J25, 42C99.

Key words and phrases: families of operators, isomorphic domains, boundary conditions, eigenspaces, Fourier series.

Research of T. Winiarski partially supported by KBN grant no. 2 P03A 01522. 
In Section 2.2 there is a construction of a continuous family $\Psi_{t}$ for some families $\left(\mathcal{D}_{t}\right)_{t \in \mathcal{T}}$ of domains of operators in a Hilbert space $H$ for which the corresponding eigenspaces form a complete orthogonal system of closed linear subspaces of $H$.

If $\mathcal{D}_{t}$ is the domain of a closed invertible operator $A_{t}: X \rightarrow X$ and $R_{t}=$ $A_{t}^{-1}$, for $t \in \mathcal{T}$, then the natural candidate for $\Phi_{t}=\Psi_{t}^{-1}$ is $\overline{R_{t_{0}} A_{t}}$ whenever $R_{t_{0}} A_{t}$ is closable. If it is closable then we may use some results presented in [3] concerning the topology of generalized convergence to prove that the expected family is good (for more details see Section 3). Unfortunately, if $\mathcal{D}_{t}$ depends on $t$, it may happen that $R_{t_{0}} A_{t}$ is not closable.

2. Regularity of families of linear subspaces. Let $X$ be a Banach space, $\mathcal{T}$ an interval in $\mathbb{R}$, and $\left(\mathcal{D}_{t}\right)_{t \in \mathcal{T}}$ a family of linear subspaces of $X$.

Definition 1. We say that the family $\left(\mathcal{D}_{t}\right)_{t \in \mathcal{T}}$ is of class $\mathcal{C}_{a}{ }^{k}$ (resp. strongly of class $\mathcal{C}_{a}{ }^{k}$ ) if there exist a linear subspace $\mathcal{D}$ of $X$ and a family $\left(\Psi_{t}\right)_{t \in \mathcal{T}}$ of automorphisms of $X$ such that

- the mapping $\mathcal{T} \ni t \mapsto \Psi_{t} \in \operatorname{Aut}(X)$ is of class $\mathcal{C}^{k}$ (resp. strongly of class $\mathcal{C}^{k}\left({ }^{1}\right)$ and

- $\Psi_{t}(\mathcal{D})=\mathcal{D}_{t}$ for $t \in \mathcal{T}$.

Considering a family $\left(A_{t}\right)_{t \in \mathcal{T}}$ of closed linear operators with the family of domains $\left(\mathcal{D}_{t}=\mathcal{D}\left(A_{t}\right)\right)_{t \in \mathcal{T}}$ of class $\mathcal{C}_{a}{ }^{k}$ we may reduce some problems to a family with a constant domain. For example, suppose that $u$ is a classical solution of the evolution equation

$$
\frac{d u}{d t}=A(t) u+f(t)
$$

in which the family of domains $\left(\mathcal{D}_{t}=\mathcal{D}\left(A_{t}\right)=\mathcal{D}(A(t))\right)_{t \in \mathcal{T}}$ is of class $\mathcal{C}_{a}{ }^{1}$. Let $\left(\Psi_{t}\right)_{t \in \mathcal{T}}$ be a family of automorphisms of $X$ as above and $\left(\Phi_{t}=\Psi_{t}^{-1}\right)_{t \in \mathcal{T}}$ the family of inverses.

Since $u(t) \in \mathcal{D}_{t}$, there exists $v(t) \in \mathcal{D}$ such that $\Psi_{t}(v(t))=u(t)$. We have

$$
\frac{d u}{d t}=\frac{d \Psi_{t}}{d t} v(t)+\Psi_{t} \frac{d v}{d t}
$$

and after a standard calculation we obtain

$$
\frac{d v}{d t}=\underbrace{\left(\Phi_{t} A(t) \psi_{t}-\frac{d \psi_{t}}{d t}\right)}_{B(t)} v(t)+\underbrace{\Phi_{t} f(t)}_{F(t)} .
$$

Thus $v$ is a classical solution of the evolution equation

$$
\frac{d v}{d t}=B(t) v+F(t)
$$

with the family $\left(B_{t}=B(t)\right)_{t \in \mathcal{T}}$ of operators having domains independent of $t$.

$\left({ }^{1}\right)$ This means that for any $x \in X$ the mapping $t \mapsto \Psi_{t} x$ is of class $\mathcal{C}^{k}$. 
2.1. Construction using boundary conditions. Now we produce an example of a family $\left(\mathcal{D}_{t}\right)_{t \in \mathcal{T}}$ of class $\mathcal{C}_{a}{ }^{k}, k \geq 1$, of the domains for elliptic operators of order two which is a nonconstant family of linear subspaces of $X=\mathcal{L}^{2}(\Omega)$.

Let $\Omega$ be a bounded domain in $\mathbb{R}^{n}$ with boundary $S=\partial \Omega$ of class $\mathcal{C}^{k+1}$, $\mathcal{T}=[0, T]$, and let $a: \bar{\Omega} \times \mathcal{T} \rightarrow \mathbb{R}$ be a function of class $\mathcal{C}^{k}$ nonvanishing on $S$. The sets

$$
\begin{aligned}
& \mathcal{D}_{t}=\left\{u \in \mathcal{L}^{2}(\Omega): u \in H^{2}(\Omega) \text { and } \frac{\partial u}{\partial n}+a(x, t) u=0 \text { on } \partial \Omega\right\}, \\
& \mathcal{D}=\left\{u \in \mathcal{L}^{2}(\Omega): u \in H^{2}(\Omega) \text { and } \frac{\partial u}{\partial n}=0 \text { on } \partial \Omega\right\}
\end{aligned}
$$

are dense linear subspaces of $\mathcal{L}^{2}(\Omega)$, where $n$ is the interior unit normal vector field on $S$.

Let $\eta: \bar{\Omega} \times \mathcal{T} \rightarrow \mathbb{R}$ be a function of class $\mathcal{C}^{k}$ such that

$$
\begin{aligned}
& 1 / 2 \leq \eta_{t}(x)=\eta(x, t) \quad \text { for } x \in \bar{\Omega}, t \in \mathcal{T}, \\
& \eta_{t}(x)=1 \quad \text { and } \quad \frac{\partial \eta_{t}(x)}{\partial n}=a(x, t) \quad \text { for } x \in \partial \Omega, t \in \mathcal{T} .
\end{aligned}
$$

The function $\eta$ can be constructed in the following way. We consider $S$ as the retract of class $\mathcal{C}^{k}$ (for $\varepsilon>0$ small enough) of the open $\varepsilon$-tube

$$
\operatorname{TUB}^{\varepsilon}(S)=\{x+\tau n(x): x \in S,|\tau|<\varepsilon\} .
$$

Then we take a function $h_{\varepsilon}$ of class $\mathcal{C}^{\infty}$ in $\mathbb{R}^{n}$ satisfying the following conditions:

$$
\begin{array}{ll}
h_{\varepsilon}(x)=0 & \text { for } x \in \mathbb{R}^{n} \backslash \operatorname{TUB}^{\varepsilon}(S), \\
h_{\varepsilon}(x)=1 & \text { for } x \in \operatorname{TUB}^{\varepsilon / 2}(S), \\
h_{\varepsilon}(x) \in[0,1] & \text { for } x \in \mathbb{R}^{n} .
\end{array}
$$

The function

$$
f_{\varepsilon}: \operatorname{TUB}^{\varepsilon}(S) \ni x+\tau n(x) \mapsto a(t, x) \tau \in \mathbb{R}
$$

is of class $\mathcal{C}^{k}$, and for $\varepsilon$ small enough, the function $\eta=h_{\varepsilon} f_{\varepsilon}+1$ is one we have been looking for.

Let $\Phi_{t}: \mathcal{L}^{2}(\Omega) \rightarrow \mathcal{L}^{2}(\Omega)$ be given by

$$
\Phi_{t}(u)=\eta_{t} \cdot u \quad \text { for } u \in \mathcal{L}^{2}(\Omega), t \in[0, T]
$$

and let $\Psi_{t}=\Phi_{t}^{-1}$. One can verify that

- $\Phi_{t} \in \operatorname{Aut}\left(\mathcal{L}^{2}(\Omega)\right)$,

- $\Phi_{t}\left(\mathcal{D}_{t}\right)=\mathcal{D}$ and $\Psi_{t}(\mathcal{D})=\mathcal{D}_{t}$,

- the mapping $\mathcal{T} \ni t \mapsto \Phi_{t} \in \mathcal{B}\left(\mathcal{L}^{2}(\Omega)\right)$ is of class $\mathcal{C}^{k}$. Thus the mapping $\mathcal{T} \ni t \rightarrow \Psi_{t} \in \mathcal{B}\left(\mathcal{L}^{2}(\Omega)\right)$ is also of class $\mathcal{C}^{k}$. 
Considering parametrized boundary conditions of the form

$$
\frac{\partial u}{\partial \mu_{t}}+a(x, t) u=0 \quad \text { on } \partial \Omega,
$$

where $\mu_{t}$ is a vector field on $S$ parametrized by $t \in \mathcal{T}$, one can look for $\Phi_{t}$ of the form

$$
\Phi_{t} u=\eta_{t} \cdot\left(u \circ \varphi_{t}\right) \quad \text { for } u \in \mathcal{L}^{2}(\Omega),
$$

where $\varphi_{t}$ is a diffeomorphism of $\bar{\Omega}$ such that $\varphi_{t}^{\prime}(x) \cdot n(x)=\mu_{t}(x), \varphi_{t}(x)=x$ for $x \in S, t \in \mathcal{T}$, and $\eta$ is as in (8). Indeed, if $u$ satisfies (9) then

$$
\frac{\partial(u \circ \varphi)}{\partial n}(x)+a(x, t)(u \circ \varphi)(x)=\frac{\partial u}{\partial \mu_{t}}+a(x, t) u=0 \quad \text { on } \partial \Omega .
$$

Thus, $v=u \circ \varphi_{t} \in \mathcal{D}_{t}, \eta_{t} v \in \mathcal{D}$ and vice versa.

Let us remark that the boundary conditions (9) parametrized by $t$ are natural, for example, when we consider the family $\left(A_{t}=t A+(1-t) \Delta\right)_{t \in[0,1]}$ in which $A$ is a strongly elliptic operator of the second order. For an application see the second part of the proof of Theorem 3.4 in [1].

2.2. Construction using eigenspaces. Let $H$ be a Hilbert space, and $H_{j}$, $j=1,2, \ldots$, a complete orthogonal sequence of closed linear subspaces of $H$. We will use the following well known facts from the theory of Fourier series.

Lemma 1. If $a_{j} \in H_{j}$ for $j=1,2, \ldots$ then the series $\sum_{j=1}^{\infty} a_{j}$ converges to a point $a \in H$ if and only if the series $\sum_{j=1}^{\infty}\left\|a_{j}\right\|^{2}$ is convergent. Moreover, if

$$
a=\sum_{j=1}^{\infty} a_{j}
$$

then

$$
\|a\|^{2}=\sum_{j=1}^{\infty}\left\|a_{j}\right\|^{2} \quad \text { and } \quad a_{j}=p_{j}(a) \text { for } j=1,2, \ldots,
$$

where $p_{j}: H \rightarrow H_{j}$ is the orthogonal projection of $H$ onto $H_{j}$ for $j=$ $1,2, \ldots$

To any sequence $\lambda=\left\{\lambda_{j}\right\}_{j=1}^{\infty}$ of real (complex if $H$ is a complex space) numbers corresponds a closed linear operator $A=A_{\lambda}(t): H \rightarrow H$ given by

$$
A x=A_{\lambda} x=\sum_{j=1}^{\infty} \lambda_{j} p_{j}(x) .
$$

The operator $A$ with domain

$$
\mathcal{D}:=\mathcal{D}(A)=\left\{x \in H: \sum_{j=1}^{\infty} \lambda_{j} p_{j}(x) \text { is convergent }\right\}
$$


is a closed densely defined linear operator and $\lambda_{j}$ is an eigenvalue of $A$ corresponding to the eigenspace $H_{j}$.

From now on we assume that $H_{j}$ and $\lambda_{j}$ depend on the parameter $t \in \mathcal{T}$. This implies that the projections $p_{j}, j=1,2, \ldots$, also depend on $t$. Thus, $H_{j}(t), \lambda_{j}(t), p_{j}(t), j=1,2, \ldots$, are sequences of closed subspaces, numbers and projections, respectively, parametrized by $t \in \mathcal{T}$.

Proposition 2. Suppose that for given $t, t_{0} \in \mathcal{T}, \Phi_{j}(t): H \rightarrow H$, $j=1,2, \ldots$, are bounded linear mappings satisfying the following conditions:

(i) $\Phi_{j}(t)\left(H_{j}(t)\right)=H_{j}\left(t_{0}\right)$ and $\left.\Phi_{j}(t)\right|_{H_{j}(t)}: H_{j}(t) \rightarrow H_{j}\left(t_{0}\right)$ is an isomorphism of Banach spaces for $j=1,2, \ldots$,

(ii) there exist positive constants $M(t), m(t)>0$ such that

$$
m(t)\|x\| \leq\left\|\Phi_{j}(t) x\right\| \leq M(t)\|x\| \quad \text { for } x \in H_{j}(t), j=1,2, \ldots,
$$

(iii) there exist positive constants $\delta(t), \Delta(t)>0$ such that

$$
\delta(t) \leq\left|\frac{\lambda_{j}\left(t_{0}\right)}{\lambda_{j}(t)}\right| \leq \Delta(t) \quad \text { for } j=1,2, \ldots
$$

Then

$$
\Phi_{t}:=\Phi(t)=\sum_{j=1}^{\infty} \Phi_{j}(t) \circ p_{j}(t)
$$

is an automorphism of $H$ such that $\Phi_{t}\left(\mathcal{D}_{t}\right)=\mathcal{D}_{t_{0}}$.

Proof. We begin by proving that $\Phi_{t}$ is well defined. Since

$$
\left\|\Phi_{j}(t)\left(p_{j}(t) x\right)\right\|^{2} \leq\left\|\Phi_{j}(t)\right\|^{2}\left\|p_{j}(t) x\right\|^{2} \leq M^{2}(t)\left\|p_{j}(t) x\right\|^{2}
$$

and the series $\sum_{j=1}^{\infty}\left\|p_{j}(t) x\right\|^{2}$ is convergent (because $\sum_{j=1}^{\infty} p_{j}(t) x$ is convergent), the series defining $\Phi_{t} x$ is convergent for any $(t, x) \in I \times H$.

Since

$$
\left\|\Phi_{t} x\right\|^{2}=\sum_{j=1}^{\infty}\left\|\Phi_{j}(t) p_{j}(t) x\right\|^{2} \leq M^{2}(t)\|x\|^{2},
$$

the operator $\Phi_{t}$ is bounded.

Injectivity of $\Phi_{t}$ follows from Lemma 1. Indeed,

$$
\operatorname{ker} \Phi_{t}=\left\{x \in H: \Phi_{j}(t) p_{j}(t) x=0, j=1,2, \ldots\right\}=\{0\} .
$$

Let $y \in H$ and

$$
x=\sum_{j=1}^{\infty}\left(\Phi_{j}(t)\right)^{-1} p_{j}\left(t_{0}\right) y .
$$

To prove surjectivity we must prove that the series (12) defining $x$ is convergent and that $\Phi_{t} x=y$. 
Assuming the convergence for the moment, we have

$$
\Phi_{t} x=\sum_{j=1}^{\infty} \Phi_{j}(t) p_{j}(t) x=\sum_{j=1}^{\infty} \Phi_{j}(t)\left(\Phi_{j}(t)\right)^{-1} p_{j}\left(t_{0}\right) y=\sum_{j=1}^{\infty} p_{j}\left(t_{0}\right) y=y .
$$

The convergence of $\sum_{j=1}^{\infty}\left(\Phi_{j}(t)\right)^{-1} p_{j}\left(t_{0}\right) y$ follows from Lemma 1 , because of the estimates

$$
\left\|\left(\Phi_{j}(t)\right)^{-1} p_{j}\left(t_{0}\right) y\right\|^{2} \leq \frac{1}{m(t)}\left\|p_{j}(t) y\right\|^{2} \quad \text { for } j=1,2, \ldots, y \in X .
$$

For $x \in \mathcal{D}_{t}$ the series $\sum_{j=1}^{\infty} \lambda_{j}(t) p_{j}(t) x$ is convergent and we have $\Phi_{t} x=y=$ $\sum_{j=1}^{\infty} p_{j}\left(t_{0}\right) y$. Thus

$$
\sum_{j=1}^{\infty} \Phi_{j}(t) p_{j}(t) x=\sum_{j=1}^{\infty} p_{j}\left(t_{0}\right) y
$$

which implies that

$$
p_{j}\left(t_{0}\right) y=\Phi_{j}(t) p_{j}(t) x
$$

Since

$$
\begin{aligned}
\left\|\frac{\lambda_{j}\left(t_{0}\right)}{\lambda_{j}(t)} \Phi_{j}(t)\left(\lambda_{j}(t) p_{j}(t)\right) x\right\|^{2} & \leq\left|\frac{\lambda_{j}\left(t_{0}\right)}{\lambda_{j}(t)}\right|^{2} M^{2}(t)\left\|\lambda_{j}(t) p_{j}(t)\right\|^{2} \\
& \leq \Delta^{2}(t) M^{2}(t)\left\|\lambda_{j}(t) p_{j}(t)\right\|^{2}
\end{aligned}
$$

the series

$$
\sum_{j=1}^{\infty} \frac{\lambda_{j}\left(t_{0}\right)}{\lambda_{j}(t)} \Phi_{j}(t)\left(\lambda_{j}(t) p_{j}(t)\right) x
$$

is convergent and hence, because of (13), so is $\sum_{j=1}^{\infty} \lambda_{j}\left(t_{0}\right) p_{j}\left(t_{0}\right) y$. This means that $\Phi_{t}\left(\mathcal{D}_{t}\right) \subset \mathcal{D}_{t_{0}}$. The proof of the inverse inclusion is similar.

Remark 1. If, in Proposition 2, $\Phi_{j}(t): H_{j}(t) \rightarrow H_{j}\left(t_{0}\right)$ is an isometry for all $j$ and $t$, then $\Phi_{t}$ is also an isometry.

THEOREM 3. If the mappings

$$
I \ni t \mapsto p_{j}(t) \quad \text { and } \quad I \ni t \mapsto \Phi_{j}(t) \quad \text { for } j=1,2, \ldots
$$

are continuous and there exist $M, m>0$ such that

$$
m\|x\| \leq\left\|\Phi_{j}(t) x\right\| \leq M\|x\| \quad \text { for } j=1,2, \ldots, x \in X,
$$

then for any compact set $K \subset I \times H$, the mapping $K \ni(t, x) \mapsto \Phi_{t} x$ is continuous.

Proof. By Dini's theorem, the sequence

$$
S_{\nu}(t, x)=\sum_{j=1}^{\nu}\left\|p_{j}(t) x\right\|^{2}, \quad \nu=1,2, \ldots,
$$


converges uniformly to $\|x\|^{2}$ on compact subsets of $I \times H$. Since

$$
\left\|\sum_{j=p}^{p+s} \Phi_{j}(t)\left(p_{j}(t) x\right)\right\|^{2} \leq M^{2} \sum_{j=p}^{p+s}\left\|p_{j}(t) x\right\|^{2},
$$

the Cauchy condition of uniform convergence is satisfied for the series $\sum_{j=1}^{\infty} \Phi_{j}(t)\left(p_{j}(t) x\right)$ and so $\Phi$ is continuous on $K$.

To obtain a higher regularity for the family $\left(\Phi_{t}\right)$ we must assume a higher regularity for $\Phi_{j}(t)$ and some assumptions that guarantee differentiability of series term by term.

EXAMPLE 1. The mapping

$$
\Phi_{j}(t):=p_{j}\left(t_{0}\right) \circ p_{j}(t): H \rightarrow H_{j}\left(t_{0}\right) \subset H
$$

is a bounded linear map. Assuming, for example, that

$$
H_{j}^{\perp}\left(t_{0}\right) \cap H_{j}(t)=\{0\}
$$

we see that $\Phi_{j}(t)$ is injective.

If additionally we assume that $\operatorname{dim} H_{j}(t)=k_{j}<\infty$ is independent of $t$ then

$$
\left.\Phi_{j}(t)\right|_{H_{j}(t)}: H_{j}(t) \rightarrow H_{j}\left(t_{0}\right)
$$

is an isomorphism. Moreover

$$
\left\|\Phi_{j}(t) x\right\|^{2} \leq\left\|p_{j}(t) x\right\|^{2} \quad \text { and } \quad \Phi_{j}\left(t_{0}\right)=p_{j}\left(t_{0}\right)
$$

Therefore, using the same method as in the proof of Theorem 3, we may prove that the continuity of the mapping $I \times H \ni(t, x) \mapsto p_{j}(t) x \in H$ for $j=1,2, \ldots$ implies the continuity of $\Phi$ on compact subsets of $I \times H$.

3. Families of closed operators with bounded inverses. Let $X, Y$ be Banach spaces over the field $\mathbb{K}$ of real or complex numbers. We endow the space $C(X, Y)$ of closed linear operators $A: X \rightarrow Y$ with the topology of generalized convergence $[3, \mathrm{Ch}$. IV]. The domain of a given operator $A: X \rightarrow Y$ is denoted by $\mathcal{D}(A)$. The space of bounded linear operators $A: X \rightarrow Y$ is denoted by $\mathcal{B}(X, Y)$, and $\operatorname{Isom}(X, Y)$ is the subspace of $\mathcal{B}(X, Y)$ of bijective bounded linear operators with bounded inverses. The subspace of $C(X, Y)$ consisting of the invertible densely defined operators $A$ such that $A^{-1} \in \mathcal{B}(Y, X)$ will be denoted by $\mathcal{R}(X, Y)$. If $X=Y$ we will write $C(X), \mathcal{B}(X), \operatorname{Aut}(X), \mathcal{R}(X)$ instead of $C(X, X), \mathcal{B}(X, X), \operatorname{Isom}(X, X)$, $\mathcal{R}(X, X)$, respectively. Since $\mathcal{B}(X, Y) \subset C(X, Y)$, we may consider $\mathcal{B}(X, Y)$ with the induced topology, which by [3, Ch. IV, Theorem 2.23] is equivalent to the norm topology in $\mathcal{B}(X, Y)$. Let us also recall that by the same theorem, the convergence of $A_{n}$ to $A$ in $\mathcal{R}(X, Y)$ is equivalent to the convergence of $A_{n}^{-1}$ to $A^{-1}$ in $\mathcal{B}(Y, X), \operatorname{Isom}(X, Y)$ is open in $C(X, Y)$ and $\operatorname{Aut}(X)$ is open in $C(X)$. 
Lemma 4. Let $\mathcal{H}$ be a metric space, $A_{h} \in \mathcal{R}(X, Y)$ and $\Phi_{h} \in \operatorname{Aut}(X)$ for $h \in \mathcal{H}$. If the mappings

$$
\mathcal{H} \ni h \mapsto A_{h} \in C(X, Y) \quad \text { and } \quad \mathcal{H} \ni h \mapsto \Phi_{h} \in \mathcal{B}(X)
$$

are continuous then the mapping

$$
\mathcal{H} \ni h \mapsto A_{h} \circ \Phi_{h} \in \mathcal{R}(X, Y)
$$

is also continuous.

Proof. Since $A_{h} \in \mathcal{R}(X, Y)$, the continuity of $\mathcal{H} \ni h \mapsto A_{h} \in C(X, Y)$ is equivalent to the continuity of $\mathcal{H} \ni h \mapsto A_{h}^{-1} \in \mathcal{B}(Y, X)$. Thus the mapping $\mathcal{H} \ni h \mapsto\left(A_{h} \circ \Phi_{h}\right)^{-1}=\Phi_{h}^{-1} \circ A_{h}^{-1} \in \mathcal{B}(Y, X)$ is continuous, and hence so is the mapping (15).

Lemma 5. Let $A_{j} \in \mathcal{R}(X, Y)$ and $R_{j}=A_{j}^{-1}$ for $j=1,2$. If $R_{j} \circ A_{i}$ is bounded for $i, j=1,2$, then $\mathcal{D}\left(A_{1}^{*}\right)=\mathcal{D}\left(A_{2}^{*}\right)$.

Proof. By symmetry, it is enough to prove that $\mathcal{D}\left(A_{1}^{*}\right) \subset \mathcal{D}\left(A_{2}^{*}\right)$. Take $y^{*} \in \mathcal{D}\left(A_{1}^{*}\right)$. Since

$$
\begin{aligned}
\left|\left\langle A_{2} x, y^{*}\right\rangle\right| & =\left|\left\langle A_{1} \circ\left(R_{1} \circ A_{2}\right) x, y^{*}\right\rangle\right|=\left|\left\langle\left(R_{1} \circ A_{2}\right) x, A_{1}^{*} y^{*}\right\rangle\right| \\
& \leq\left\|A_{1}^{*} y^{*}\right\| \cdot\left\|R_{1} \circ A_{2}\right\| \cdot\|x\| \quad \text { for } x \in \mathcal{D}\left(A_{2}\right),
\end{aligned}
$$

we have $y^{*} \in \mathcal{D}\left(A_{2}^{*}\right)$ and so $\mathcal{D}\left(A_{1}^{*}\right) \subset \mathcal{D}\left(A_{2}^{*}\right)$.

Lemma 6. If $A \in C(X, Y)$ and $\Phi \in \mathcal{B}(X)$ is such that $\mathcal{D}(A \circ \Phi)$ is dense in $X$ then $\mathcal{D}\left(A^{*}\right) \subset \mathcal{D}\left((A \circ \Phi)^{*}\right)$. Moreover, if $\Phi \in \operatorname{Aut}(X)$ then $\mathcal{D}\left(A^{*}\right)=\mathcal{D}\left((A \circ \Phi)^{*}\right)$.

Proof. Let $y^{*} \in \mathcal{D}\left(A^{*}\right)$. Since $\Phi$ is continuous, for $x \in \mathcal{D}(A \circ \Phi)$ we have

$$
\left|\left\langle(A \circ \Phi) x, y^{*}\right\rangle\right|=\left|\left\langle\Phi x, A^{*} y^{*}\right\rangle\right| \leq\left\|A^{*} y^{*}\right\| \cdot\|\Phi x\| \leq\left\|A^{*} y^{*}\right\| \cdot\|\Phi\| \cdot\|x\|
$$
and so $y^{*} \in \mathcal{D}\left((A \circ \Phi)^{*}\right)$.

If $\Phi$ is invertible then by the above $\mathcal{D}\left((A \circ \Phi)^{*}\right) \subset \mathcal{D}\left(\left(A \circ \Phi \circ \Phi^{-1}\right)^{*}\right)=$ $\mathcal{D}\left(A^{*}\right)$.

Theorem 7. Let $(\mathcal{H}, \varrho)$ be a connected metric space and $\left(A_{h}\right)_{h \in \mathcal{H}}$ a family of linear operators $A_{h} \in \mathcal{R}(X, Y)$. If $R_{k} \circ A_{h}$ is closable for each $h, k \in \mathcal{H}$, and for each $k \in \mathcal{H}$ the mapping

$$
\mathcal{H} \ni h \mapsto \overline{R_{k} \circ A_{h}} \in C(X)
$$

is continuous, then:

(i) $\overline{R_{k} \circ A_{h}} \in \operatorname{Aut}(X)$ for each $h, k \in \mathcal{H}$,

(ii) for any $h, k \in \mathcal{H}$ there exist $m, M>0$ such that

$$
m\left\|R_{h} y\right\| \leq\left\|R_{k} y\right\| \leq M\left\|R_{h} y\right\| \quad \text { for } y \in Y,
$$

(iii) $\mathcal{D}\left(A_{h}^{*}\right)=\mathcal{D}^{*}=$ const,

(iv) $\mathcal{D}\left(A_{h}^{*} \circ R_{k}^{*}\right)=X^{*}$ for all $h, k \in \mathcal{H}$. 
Proof. Since $\operatorname{Aut}(X)$ is open in $C(X)$ and $\overline{R_{k} \circ A_{k}}=\operatorname{Id}_{X} \in \operatorname{Aut}(X)$, there exists $\delta=\delta(k)>0$ such that $\overline{R_{k} \circ A_{h}} \in \operatorname{Aut}(X)$ for any $h \in \mathcal{H}$ such that $\varrho(h, k)<\delta$. Thus, for a given $k \in \mathcal{H}$,

$$
\mathfrak{M}=\left\{h \in \mathcal{H}: \overline{R_{k} \circ A_{h}} \in \operatorname{Aut}(X)\right\} \neq \emptyset .
$$

To prove that $\mathfrak{M}=\mathcal{H}$ it is enough to prove that $\mathfrak{M}$ is open and closed. For given $h_{0} \in \mathfrak{M}, h \in \mathcal{H}$ we have

$$
\overline{R_{k} \circ A_{h}}=\overline{R_{k} \circ A_{h_{0}}} \circ \overline{R_{h_{0}} \circ A_{h}} .
$$

Since $\overline{R_{k} \circ A_{h_{0}}} \in \operatorname{Aut}(X)$, and by the same argument as before there exists $\delta=\delta\left(h_{0}\right)>0$ such that $\overline{R_{h_{0}} \circ A_{h}} \in \operatorname{Aut}(X)$ for any $h \in \mathcal{H}$ satisfying $\varrho\left(h, h_{0}\right)<\delta$, the set $\mathfrak{M}$ is open. Suppose now that $h_{n} \in \mathfrak{M}$ for $n=1,2, \ldots$ and $h_{n} \rightarrow h_{0} \in \mathcal{H}$ as $n \rightarrow \infty$. Then there exists $n \in \mathbb{N}$ such that $\overline{R_{h_{0}} \circ A_{h_{n}}} \in$ $\operatorname{Aut}(X)$, by the previous part of the proof. Since $\overline{R_{k} \circ A_{h_{n}}}=\overline{R_{k} \circ A_{h_{0}}}$ 。 $\overline{R_{h_{0}} \circ A_{h_{n}}}$ and $\overline{R_{k} \circ A_{h_{n}}}, \overline{R_{h_{0}} \circ A_{h_{n}}}$ are automorphisms of $X$, it follows that $h_{0} \in \mathfrak{M}$ and so $\mathfrak{M}$ is closed.

To prove (ii) fix $h, k \in \mathcal{H}$. Since $\overline{R_{k} \circ A_{h}} \in \operatorname{Aut}(X)$, there exist $m, M>0$ such that

$$
m\|x\| \leq\left\|\left(R_{k} \circ A_{h}\right) x\right\| \leq M\|x\| \quad \text { for } x \in \mathcal{D}\left(A_{h}\right) .
$$

Since $A_{h}$ is onto, taking $y=A_{h} x$ we get

$$
m\left\|R_{h} y\right\| \leq\left\|R_{k} y\right\| \leq M\left\|R_{h} y\right\| \quad \text { for } y \in Y .
$$

To prove (iii) observe that for $k, h \in \mathcal{H}$ we have $A_{h}=A_{k} \circ \overline{R_{k} \circ A_{h}}$. Thus, by Lemma $6, \mathcal{D}\left(A_{h}^{*}\right)=\mathcal{D}\left(A_{k}^{*}\right)$, because $\overline{R_{k} \circ A_{h}} \in \operatorname{Aut}(X)$.

(iv) is a consequence of the fact that $R_{k}^{*} \in \mathcal{B}\left(X^{*}, Y^{*}\right)$ is the inverse to $A_{k}^{*}$ (see e.g. [3, Ch. III, Theorem 5.30]), which has the same domain as $A_{h}^{*}$, because of (3).

REMARK 2. Observe that for $h, k \in \mathcal{H}$, if $R_{k} \circ A_{h}$ and $R_{h} \circ A_{k}$ are closable then conditions (i)-(iv) of Theorem 7 are equivalent. If $\mathcal{D}\left(A_{h}^{*} \circ R_{k}^{*}\right)$ is dense in $X^{*}$ in the weak* topology on $X^{*}$ then $R_{k} \circ A_{h}$ is closable. If condition (iii) of Theorem 7 is satisfied, then $R_{k} \circ A_{h}$ is closable and (i), (ii), (iv) hold.

A sufficient condition for the assumptions of Theorem 7 to hold is presented in the following

Proposition 8. If $\mathcal{H}=[0, T]$, all the operators of the family $\left(A_{t}^{*}\right)_{t \in[0, T]}$ have the same domain $\mathcal{D}^{*}$ and for every $y^{*} \in \mathcal{D}^{*}$ the mapping

$$
[0, T] \ni t \mapsto A_{t}^{*} y^{*} \in X^{*}
$$

is of class $\mathcal{C}^{1}$ then the family $\left(A_{t}\right)_{t \in[0, T]}$ satisfies the assumptions of Theorem 7 .

Proof. By [4, Ch. II, Lemma 1.5], the family $\left(A_{t}^{*} \circ R_{s}^{*}\right)_{s, t \in[0, T]}$ of bounded operators is continuous with respect to $(s, t)$. Since also $A_{t}^{*} \circ R_{s}^{*}=\left(R_{s} \circ A_{t}\right)^{*}$ 
and $\mathcal{D}\left(\left(R_{s} \circ A_{t}\right)^{*}\right)=X^{*}$, the mapping $R_{s} \circ A_{t}$ is closable, and by [3, Ch. IV, Theorem 2.23], the continuity of the family $\left(R_{s} \circ A_{t}\right)^{*}$ with respect to $(s, t)$ implies the continuity of $\left(\overline{R_{s} \circ A_{t}}\right)$.

3.1. Some remarks on the case of differential operators. Let $\Omega$ be a bounded domain in $\mathbb{R}^{n}$ with smooth boundary $\partial \Omega$, and $\mathcal{H}$ a connected metric space. Let

$$
A_{h}=\sum_{|\alpha| \leq m} a_{\alpha}(x, h) D^{\alpha} \quad \text { for } h \in \mathcal{H}
$$

be a family of differential operators of order $m$ with coefficients $a_{\alpha}$ continuous in $\bar{\Omega} \times \mathcal{H}$. Closedness of $A_{h}$ and continuity of the mapping $h \mapsto A_{h}$ depend on the domain $\mathcal{D}\left(A_{h}\right)$, the space $X$ in which $\mathcal{D}\left(A_{h}\right)$ is contained, and the space $Y$ of values of $A_{h}$.

- If $\mathcal{D}\left(A_{h}\right)=X=H^{m}(\Omega)$ and $Y=\mathcal{L}^{2}(\Omega)$ then $A_{h}$ is bounded and the mapping $\mathcal{H} \ni h \mapsto A_{h} \in \mathcal{B}(X, Y)$ is continuous.

- Let $X=Y=\mathcal{L}^{2}(\Omega)$ and let $D$ be a closed subspace of $H^{m}(\Omega)$ such that $D$ is dense in $\mathcal{L}^{2}(\Omega)$, and the mapping $A_{h}: D \rightarrow \mathcal{L}^{2}(\Omega)$ is one-to-one and onto for $h \in \mathcal{H}$. Then $R_{h}=A_{h}^{-1} \in \mathcal{B}(Y, X)$ and the mapping $\mathcal{H} \ni h \mapsto$ $A_{h} \in C(X, Y)$ is continuous. This situation often occurs when considering strongly elliptic operators $A_{h}$ with boundary operators independent of $h$,

$$
B_{j}=\sum_{|\alpha| \leq m_{j}} b_{j \alpha}(x) D^{\alpha}, \quad 1 \leq j \leq m / 2
$$

which cover $A_{h}$ for each $h \in \mathcal{H}$. If additionally we know that $\mathcal{D}\left(A_{h}^{*}\right)=\mathcal{D}^{*}$ is independent of $h$ then $R_{k} \circ A_{h}$ is closable for each $h, k$.

Now we show an example of a family $\left(\widetilde{A}_{t}\right)_{t \in \mathcal{T}}$ of elliptic operators with pairwise different domains for which the corresponding family $\left(\mathcal{D}_{t}\right)_{t \in \mathcal{T}}$ of domains is of class $\mathcal{C}_{a}{ }^{k}$ and the family of domains of the conjugate operators is independent of $t$.

Keep the notation of Section 2.1 and assume that $a(x, t)=t$. The sets $\mathcal{D}_{t}$ given by (3) are dense linear subspaces of $\mathcal{L}^{2}(\Omega)$ such that $\mathcal{D}_{t} \neq \mathcal{D}_{\tau}$ for $t \neq \tau \in[0, T]$ and $\mathcal{D}_{0}=\mathcal{D}$, where $\mathcal{D}$ is given by (4). The operator

$$
A=-\Delta+\lambda I
$$

is well defined on $H^{2}(\Omega)$; when considered as defined only on $\mathcal{D}_{t}$, it is closed, and for $\lambda$ large enough, it is onto and one-to-one. By the closed graph theorem its inverse is bounded. Let $A_{t}$ denote the operator given by (20) with domain $\mathcal{D}_{t}$.

EXAMPLE 2. The family

$$
\widetilde{A}_{t}=A_{0} \circ \Phi_{t}: \mathcal{D}_{t} \rightarrow \mathcal{L}^{2}(\Omega)
$$


parametrized by $t \in[0, T]$ is a continuous (with respect to $t$ ) family of closed densely defined linear differential operators with pairwise different domains. Indeed, since $\left(B_{t}=A_{t} \circ \Psi_{t}\right)_{t \in[0, T]}$ is a family of closed differential operators of order two with coefficients continuous with respect to both $x$ and $t$, and with domains independent of $t$, the mapping $[0, T] \ni t \mapsto B_{t} \in C\left(\mathcal{L}^{2}(\Omega)\right)$ is continuous and, by Lemma 4 , the mapping $[0, T] \ni t \mapsto A_{t}=B_{t} \circ \Phi_{t} \in$ $C\left(\mathcal{L}^{2}(\Omega)\right)$ is also continuous.

By Lemma 6 , the domain $\mathcal{D}\left(\widetilde{A}_{t}^{*}\right)=\mathcal{D}\left(A^{*}\right)$ is the same for all $t \in[0, T]$.

The next example show that in Theorem 7 the assumption of continuity of the mapping (16) cannot be replaced by the continuity of the family $\left(A_{h}\right)_{h \in \mathcal{H}}$.

ExAMPLE 3. Let $\left(A_{t}\right)_{t \in[0, T]}$ be a family of self-adjoint operators with pairwise different domains, and with the same property for the family $A_{t}^{*}$. Since $\mathcal{C}_{0}^{\infty}(\Omega) \subset \bigcap_{t \in[0, T]} \mathcal{D}_{t}, \mathcal{C}_{0}^{\infty}$ is dense in $\mathcal{L}^{2}(\Omega),\left(R_{\tau} \circ A_{t}\right) u=\left(R_{\tau} \circ A_{\tau}\right) u=$ $u$ for $u \in \mathcal{C}_{0}^{\infty}(\Omega)$ and $\left(R_{\tau} \circ A_{t}\right) u \neq u$ for $u \in D_{t} \backslash D_{\tau}$, it follows that the operator $R_{\tau} \circ A_{t}$ is not closable for $t \neq \tau$. Thus, the mapping (16) is even not well defined.

\section{References}

[1] Yu. M. Berezanskiǔ, Eigenfunction Expansions of Self-Adjoint Operators, Naukova Dumka, Kiev, 1965 (in Russian).

[2] L. Hörmander, Linear Partial Differential Operators, Springer, 1963.

[3] T. Kato, Perturbation Theory for Linear Operators, Springer, 1980.

[4] S. G. Krĕ̌n, Linear Differential Equations in Banach Spaces, Nauka, Moscow, 1963 (in Russian).

[5] J. van Neerven, The Adjoint of a Semigroup of Linear Operators, Springer, 1992.

[6] M. Schechter, Differentiability of solutions of elliptic problems with respect to parameters, Boll. Un. Mat. Ital. A 13 (1976), 601-608.

[7] T. Winiarska, Differentiability of solutions with respect to parameters, Univ. Iagell. Acta Math. 26 (1987), 217-225.

[8] —, Differential Equations with Parameter, Technical Univ. of Cracow, Monograph 68, 1988.

Teresa Winiarska

Institute of Mathematics

Technical University of Kraków

Warszawska 24

31-155 Kraków, Poland

E-mail: twiniars@usk.pk.edu.pl
Tadeusz Winiarski Institute of Mathematics Jagiellonian University Reymonta 4 30-059 Kraków, Poland E-mail: winiarsk@im.uj.edu.pl 